\title{
INVESTIGACIÓN CIENTÍFICA, SOCIEDAD Y EDUCACIÓN
}

\section{UNA VISIÓN SOCIOHISTÓRICA DESDE LA MODERNIDAD}

\author{
Jaime Ríos Burga*
}

\section{Resumen}

El presente artículo busca destacar algunos elementos centrales de los paradigmas de la investigación científica en la vida moderna y su impacto la sociedad y educación en el mundo global de hoy.

\section{Abstrac}

This article highlights some key elements of the paradigms of scientific research in modern life and its impact society and education in the global world today.

\section{Ubicación de la problemática}

La relación entre ciencia y sociedad en la modernidad va de la mano de cada una de sus etapas de revolución industrial. Un proceso que hoy responde a nuevas preguntas como: ¿Por qué hoy el diálogo trans y multidisciplinario de la ciencia sustituye la visión y enfoque disciplinar y subdisciplinar? ¿Cómo el nuevo paradigma cientííco social afecta la investigación e institucionalidad científica educativa? ¿Cuál es su impacto en la formación y el ejercicio profesional en un mercado capitalista cada vez más global? ¿Cuáles son sus limitaciones frente al permanente cambio e innovación científico técnica pos digital?

Las comunidades científicas producto de los entornos globales construyen sus modelos de organización científica cada vez más de manera sistémica en la unidad de la diversidad de la ciencia y la tecnología estrechamente vinculadas a las

\footnotetext{
* JAIME RÍOS BURGA. Dr. Ciencias Políticas y Sociología por la Universidad Complutense de Madrid, 1990. Ex Director de la E.A.P. de Sociología de FCCS de la UNMSM. 2010-20I5. Profesor principal en la E.A.P. de Sociología de la UNMSM. Profesor en el Post Grado del Instituto de la Calidad de la educación de la USMP. Miembro de la Junta Directiva de la Asociación Latinoamericana de Sociología 20 I 5-20 17 (ALAS).
} 
demandas de la vida social. La globalización actual unida al nuevo modo de desarrollo infocomunicacional y la transformación del poder mundial nos plantea como nunca antes un nuevo desafío: la unidad de las ciencias y su integración a la solución de los problemas centrales de la vida.

Si en la modernidad clásica cada ciencia como disciplina y sus subdisciplinas precisaban de campos específicos de situaciones y problemáticas. Por tanto, un tipo de actividad de construcción de conocimientos, teorías y metodologías, es decir, formas de razonar y resolver los problemas de investigación. Hoy sin negar estas delimitaciones las ciencias como disciplinas y subdisciplinas se integran al nuevo paradigma sistémico e histórico de la ciencia a partir de la investigación en un diálogo cada vez más fecundo de los problemas de la vida. Las culturas disciplinarias cerradas dan paso a una nueva cultura trans y multidisciplinaria basada en la integración teórica y aplicada de la ciencia y tecnología.

Si bien en algunos campos la estructura disciplinaria de la ciencia todavía tiene una fuerte influencia sobre todo en países de poca investigación y fuerte burocratización institucional. Las pasadas institucionalidades o comunidades científicas en sus formas organizativas entran en crisis y cambio plantando el desafío de crear nuevas estructuras de producción colectivas de conocimientos integradas como nuevas formas de organización de la ciencia, presentes en los nuevos programas de investigación transversales, trabajos de investigación y aplicación en equipos, la estabilidad de los profesores investigadores y la participación de los estudiantes creciente de los alumnos posgrado y pregrado en investigaciones de problemas relevantes de la vida, incluidas los de la vida social. ¿Cómo hemos llegado a esta situación?

\section{De la ciencia disciplinaria a la ciencia trans y multidisciplinaria: algunas cuestiones epistemológicas}

La ciencia moderna de sus etapas iniciales vinculaba el concepto de disciplina a un área o actividad de conocimiento por conocer sistemáticamente por medio de una teoría, metodología y métodos específicos. La legitimación y autoimagen disciplinaria surgía y desarrollaba así como una característica de la modernidad cientíica clásica.

En la primera revolución industrial la disciplina operaba bajo la visión irreductible de cada campo bajo una epistemología de la simplicidad y la imagen separada de las ciencias bajo dos culturas: Las ciencias naturales y las ciencias sociales. Como anota el informe de la Comisión Gulbenkian para la reestructuración de las ciencias sociales coordinado por Immanuel Wallerstein:

"La llamada visión clásica de la ciencia, que predomina desde varios siglos, fue constituida por dos premisas. Una era el modelo newtoniano en el cual hay una 
simetría entre el pasado y el futuro. Era una visión casi teológica: al igual que Dios, podemos alcanzar certezas, y por tanto no necesitamos distinguir entre pasado y el futuro puesto que todo coexiste en un presente eterno. La segunda premisa fue el dualismo cartesiano, la suposición de que existe una distinción fundamental entre la naturaleza y los humanos, entre la materia y la mente, entre el mundo físico y el mundo social/espiritual".

Una cultura tecno científica burocrática reduccionista que por preocuparse solo de la productividad separaba la estrecha relación entre las ciencias naturales, las ciencias aplicadas y las ciencias sociales presentándolas como dos campos de investigación totalmente diferenciados. Pero contradictoriamente constatamos que en su aplicación se unían en la solución de los problemas de la modernidad industrial fabril.

Vemos cómo en la modernidad industrial las ciencias se construyen desde el marco de la investigación disciplinar organizadas en comunidades científicas. Los modelos de Bacon, Galileo y Newton operaban bajo un riguroso razonamiento lógico basado a su vez en un profundo conocimiento experimental de la realidad. Esquema epistémico y metodológico que tenía que desentrañar la realidad construyendo datos a partir de experimentos con el objetivo de hallar las regularidades promedio como magnitudes medibles que nos conducen a hipótesis y a nuevos experimentos para comprobarlos y arribar a teorías y leyes.

\section{EPISTEMOLOGÍA Y METODOLOGÍA DE LA CIENCIA DISCIPLINAR}

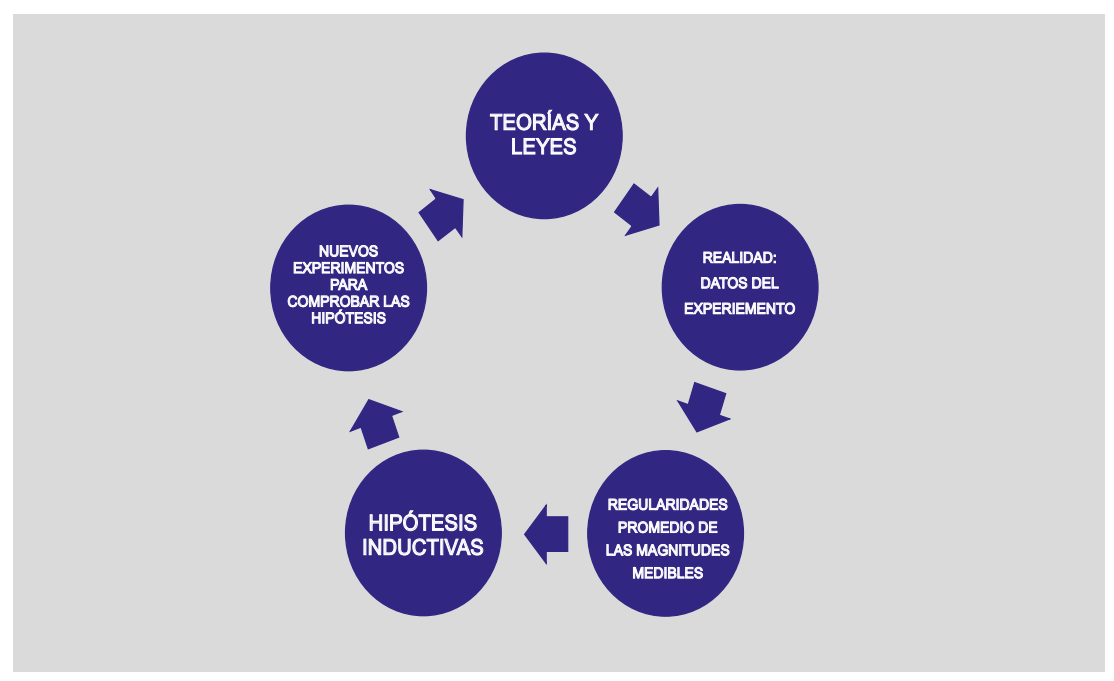

Pero las ciencias como disciplinas en sus aplicaciones fueron más allá, las organizaciones de la modernidad inicial integraron sus descubrimientos al desarrollo del capitalismo fabril en sus diferentes formas de organización. Si bien las ciencias en sus disciplinas 
y subdisciplinas tenían que afrontar su propia racionalidad de conocimiento cada vez más se vincularon en su aplicación al desarrollo del mercado mundial y los mercados nacionales.

Destaquemos solo como ejemplo, cómo a partir del descubrimiento científico de la máquina a vapor se vinculan las ciencias e impactan en la sociedad. Científicamente se integran ciencias como la química (comportamiento de los gases), la física (presión atmosférica, termodinámica), la geofísica y geología ( ubicación territorial, estructura de suelos), la ingeniería (construcción de fábricas, ferrocarriles, máquinas, desagüe de las minas), la arquitectura (urbanización industrial), la geografía (uso de los recursos naturales y humanos), la demografía (movimientos migracionales), la historia (experiencias en el tiempo), la economía (economía nacional e internacional), la sociología (integración y conflicto social), la antropología (nuevas colonizaciones), la política ( poder y política del Estado nacional), el derecho (constitución nacional).

Las ciencias tuvieron un papel clave en la revolución económica, social, política y cultural de la modernidad industrial. Su impacto fue de tal nivel que no solo transformó la economía sino creó también una nueva estructura social, política jurídica institucional y cultural capitalista. Como anota Polanyi al referirse a las consecuencias sociales de la primera revolución industrial en el mundo del trabajo:

"La separación del trabajo de otras actividades de la vida y su sometimiento a las leyes de mercado equivalió a un aniquilamiento de todas las formas orgánicas de la existencia y su sustitución por un tipo de organización diferente, atomizado e individualista. Tal plan de destrucción se vio muy bien servido por la aplicación del principio de la libertad de contrato. Esto significaba, en la práctica, que habrían de liquidarse las organizaciones no contractuales del parentesco, la vecindad, la profesión y el credo, porque reclamaban la lealtad del individuo y así restringían su libertad."

La segunda revolución industrial revoluciona la ciencia moderna procesando una mayor integración entre sus disciplinas y subdisciplinas. El modelo epistémico y metodológico de la ciencia clásica moderna se profundiza en una superespecialización donde la lógica de descubrimiento se une a la lógica de la mejor organización de la producción para el consumo. La visión del mercado influye en la centralidad de los proyectos y programas de investigación científica bajo crecientes procesos monopólicos. El capitalismo financiero estructura una nueva forma de hacer ciencia en monopolios bajo la forma de trust, cartels y holdings en el marco de los estados nación que compiten por hegemonizar el mercado mundial.

El descubrimiento de la electricidad, el petróleo, el acero, el avión, el automóvil, la bicicleta, la construcción de canales, el teléfono, etc.; revoluciona la integración 
de las ciencias, la técnica y las tecnologías como nunca antes. Ciencias que en sus disciplinas y subdisciplinas transforman la organización de la producción y el trabajo, la organización de la producción: automatización, la especialización en línea de montaje y producción a gran escala en grandes unidades monopólicas.

La revolución de la ciencia moderna como anota Javier Echevarría ha sido ampliamente estudiada por historiadores, sociólogos y filósofos. La historia, la filosofía y la sociología de la ciencia se constituyeron a principios del siglo XX y se centraron en el estudio de la ciencia moderna, incluidas las revoluciones científicas del siglo XIX y principios del XX: química (tabla periódica, química orgánica), matemáticas (Análisis, geometrías no euclídeas, teoría de conjuntos), biología (Darwin, Mendel), Geología (Lyell) y Física (Einstein, teoría cuántica).

Vemos cómo la lógica, la epistemología y la metodología de la ciencia profundiza el paradigma del análisis y reconstrucción del conocimiento científico, centrándose en los conceptos, leyes, hechos y teorías, instaurando la distinción teórico/observacional, subrayando los aspectos lógico-lingüísticos del conocimiento y desarrollando concepciones enunciativas del método científico, tanto lógico-deductivas como lógico-inductivas y estadísticas.

La mayor parte de los investigadores que se dedicaban a la investigación básica subestimaban la importancia de la tecnociencia dándole mayor importancia a los descubrimientos. Pero no habrían surgido nuevos sistemas de organización sin la integración de los diferentes campos de las ciencias unida a la técnica y la tecnología. Por ejemplo, el fordismo entre 1930-1970 creó todo un sistema de producción donde las ciencias contribuyeron a acelerar las cadenas de montaje con maquinaria especializada y un número elevado se trabajadores obreros con elevados salarios. El objetivo era tener el mayor número de productos a cada vez más a bajo costo.

Hasta la segunda revolución industrial la ciencia y la tecnología operaron de manera casi autónomas entre sí hasta la emergencia y consolidación de la tecnociencia en la tercera revolución industrial, sin perjuicio de que hayan establecido vínculos muy estrechos a lo largo sobre todo de la segunda revolución industrial. Vemos cómo en esta etapa la tecnociencia empieza a afirmarse como un campo de transformación de la vida económica y social. El motor de combustión del ingeniero Rudolf Diesel, la electricidad de Tomas Alva Edison, el telégrafo eléctrico y sin hilos de Samuel Morse y Guillermo Marconi, el cinematógrafo de los hermanos Luis y Augusto Lumiere, el aeroplano de los hermanos Montgolfier, Giffard y Wrigth entre otros inventos son la expresión de estas integraciones.

Proceso que cada vez más condicionado por el mercado mundial fue en la investigación y aplicación integrando las ciencias y las tecnologías. Como anota Pollard 
"Sobre todo fue el progreso de la tecnología, en el corazón de la industrialización, el que a un tiempo permitió y obligó al Estado a asumir funciones siempre crecientes. Papel más barato, imprenta y otros medios de comunicación, mejores métodos de transporte y de vigilancia de las fronteras aduaneras; guerra más tecnológica, que requería industrias de apoyo; una mayor proporción del comercio internacional en la renta nacional, hecha posible por un transporte más barato; una necesidad creciente de trabajadores alfabetizados en la industria; estos y otros muchos desarrollos semejantes impulsaron a los con frecuencia reacios gobiernos liberalburgueses a asumir poderes y responsabilidades cada vez más amplios."

Así, su impacto en las ciudades plantea nuevos desafíos a urbanistas y arquitectos, a la economía de mercado, la política y el papel del Estado benefactor, la gran masa de desocupados a efectos de consolidar el ejército de reserva, la consolidación de los grandes partidos políticos, las políticas públicas salariales y de seguridad social como una educación pública y privada al servicio de las nuevas modalidades de concentración y realización del capital. Etapa donde ya el trabajo científico no fue nunca solo un trabajo individual sino colectivo institucional. Si bien para el desarrollo de las ciencias la creatividad e imaginación científica cumplen un papel fundamental como motores y compromiso para el desarrollo de los descubrimientos, la vida nos muestra que la realidad económica social siempre condicionó los proyectos individuales y colectivos de investigación científica.

\section{La ciencia y la educación transcultural hoy}

En la tercera y cuarta revolución industrial el paradigma de la simplicidad cambia por el paradigma de la complejidad. La legitimación y autoimagen de cada una de las disciplinas por separado da paso a su creciente teorización y enfoque integrado en las ciencias, técnicas y tecnologías en unidad de sus diversidades. Vemos cómo las disciplinas, como conocimientos científicos específicos, se integran como interdisciplinas en un intercambio y cooperación entre sí. Así mismo, se desarrollan las pluridisciplinas como una asociación de disciplinas en virtud de proyectos u objetivos comunes. Y, vemos nacer la trans y multidisciplinas como esquemas cognitivos que atraviesan las disciplinas creando cada vez más nuevos proyectos u objetos comunes de investigación en su integración y diferencias directamente aplicadas a los mercados de producción y consumo global. 


\section{Esquema de integración de las ciencias}

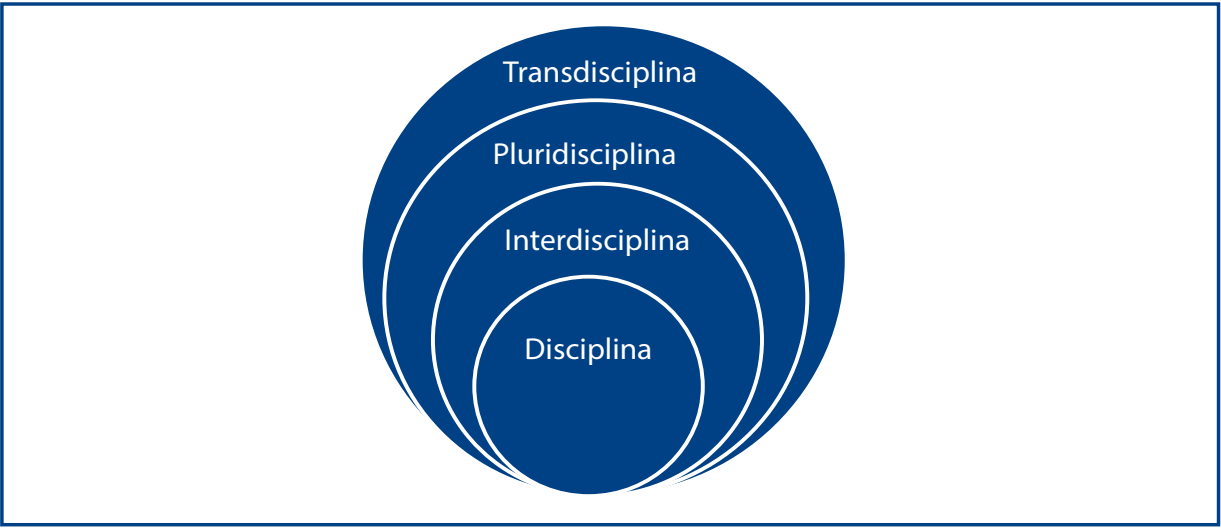

Vemos desarrollarse un paradigma diferente al de la modernidad clásica porque se construye sobre la base de una nueva epistemología y metodología creatividad reflexiva y de organización sistémica del conocimiento de la vida. Aquí el pensamiento relacional integra las ciencias de la complejidad como sistemas entrelazados en una lógica no lineal ni monocausal sino en lógicas de sistemas específicos bajo modelos y simulaciones causales complejas y no causales sino de un mundo de relaciones en interdependencias y cambios organizacionales diferenciales.

\section{Modelos de complejidad científica}

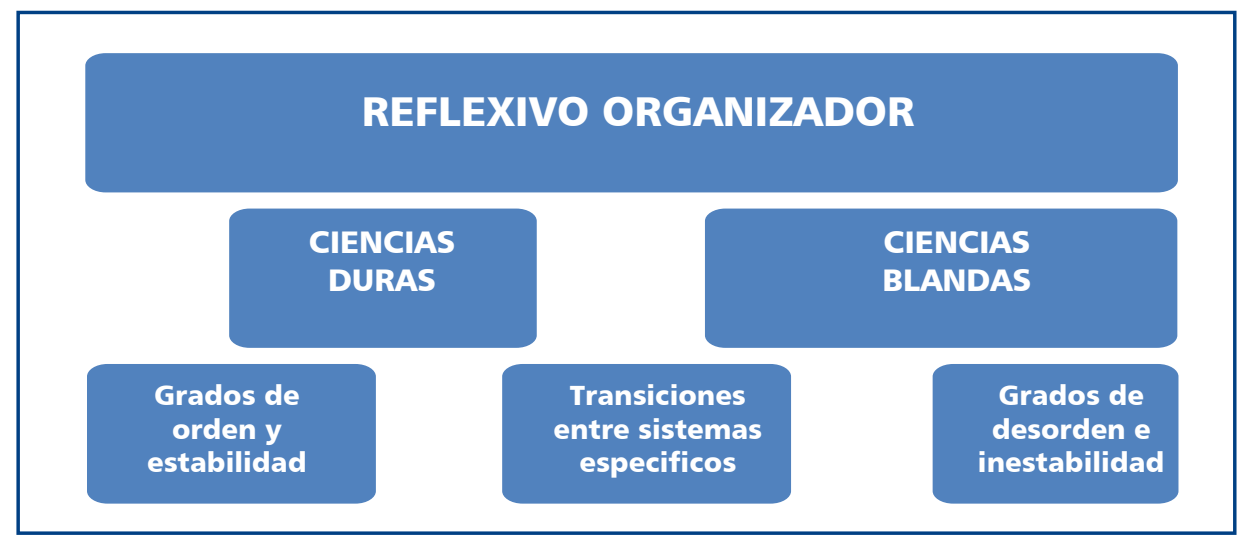

Modelos que reúnen sistemas entrelazados, sistemas específicos bajo modelos y simulaciones de unidad y diferenciación 


\section{Sistemas \\ entrelazados \\ Sistemas \\ especificos \\ Modelos y \\ simulaciones}

Modelos epistémico-metodológico y temáticos donde a diferencia del modelo clásico vinculan objeto y sujeto de conocimiento en modelos de organización complejos como sistemas históricos específicos concretos partiendo de un conocimiento relacional situacional en el marco cada vez más de metasistemas organizados como presentamos en el siguiente gráfico:

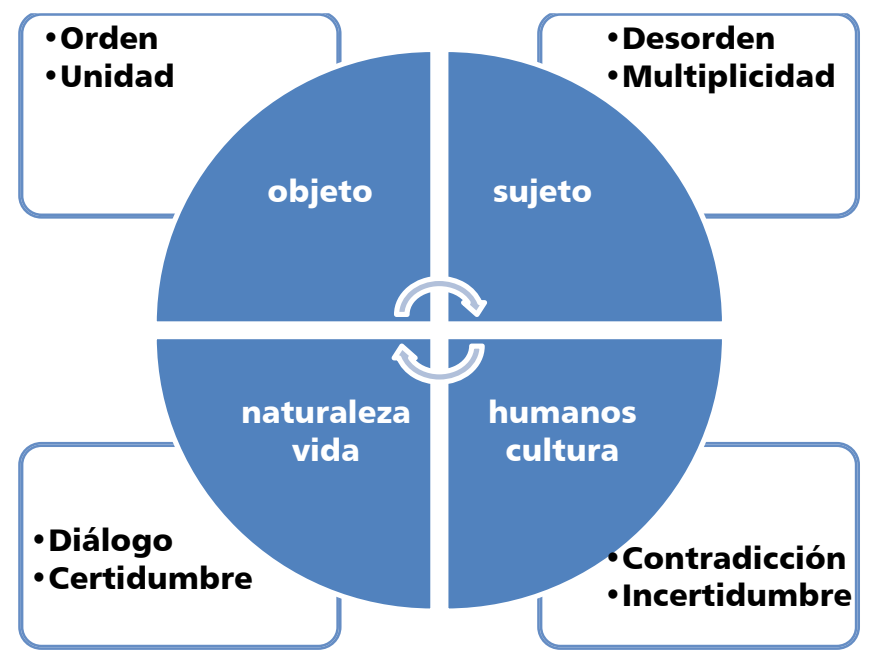

Por otra parte, la manera de investigar cambia. Como anota Echevarría:

"Si atendemos al principal escenario de la ciencia moderna, el laboratorio, la tecnociencia aporta cambios significativos. Vimos que, en el caso de la macrociencia, los laboratorios se convertían en factorías de producción de conocimiento. Con el salto ulterior a la tecnociencia, adoptan la forma de laboratorios-red, interconectados gracias a las tecnologías de la información. Frente al laboratorio aislado de la ciencia moderna, surgen los laboratorios coordinados, que colaboran en un mismo proyecto y se dividen las tareas a llevar a cabo. Otro tanto ocurre con los proyectos de investigación, en los que suelen colaborar diferentes equipos investigadores, empresas y países. En conjunto, el atomismo institucional que caracterizó a la ciencia moderna se ha visto reemplazado por una tecnociencia en red, con todas las consecuencias que ello tiene para la organización de la actividad científica y para la práctica investigadora. 
La red Arpanet, que conectó diversas universidades y agencias norteamericanas en los años 80, puede ser considerada como un primer paradigma de la investigación en red, al igual que la World Wide Web, ideada por Berners-Lee para facilitar la comunicación entre los investigadores del CERN europeo. Al laboratorio formado por el recinto físico donde coincidían presencialmente los investigadores, los aparatos y los objetos investigados se le superpuso un laboratorio-red. Los nuevos programas de investigación espacial y militar de los EEUU, completamente mediatizados por las redes telemáticas, constituyen otros dos grandes ejemplos de esta profunda transformación topológica del principal escenario donde se elaboró la ciencia moderna, el laboratorio. El acceso remoto a grandes ordenadores y equipamientos, el intercambio de datos, borradores e hipótesis a través de las redes telemáticas y la investigación en red fueron convirtiéndose a partir de los años 80 en una práctica científica habitual, sin perjuicio de que las observaciones y experimentos tradicionales siguieran desarrollándose. Los objetos investigados eran representaciones informáticas, los datos empíricos devinieron tecnodatos y los equipos de investigación y contrastación estaban dispersos geográficamente, pero conectados por vía tecnológica".

El trabajo el equipo en las ciencias naturales, la ingeniería, la medicina, las ciencias sociales, la educación, la arquitectura, las artes y las humanidades, predomina y ningún trabajo se realiza sin un conocimiento de todo lo producido anteriormente. Pero lo que marca hoy el cambio científico es el trabajo trans y multidisciplinario en equipos.

Como destaca un estudio publicado en la prestigiosa revista Science que relevó casi 20 millones de artículos científicos y 2, I millones de patentes en las últimas cinco décadas demostró que los equipos predominan sobre autores solitarios en la producción de conocimiento con alto impacto. Esto se aplica para las ciencias naturales y la ingeniería, las ciencias sociales, artes y humanidades, lo que sugiere que el proceso de creación de conocimiento ha cambiado. Sorprendentemente, este estudio encontró una tendencia igualmente fuerte hacia el trabajo en equipo en las ciencias sociales, ciencias naturales e ingeniería (de un 17,5\% en 1955 a un 51,5\% en 2000). Esto significa que se ha producido un cambio sustancial que liga la tarea de investigación a la labor colectiva. Del mismo modo, la extensión de los equipos ha ido creciendo hasta llegar a casi el doble en 45 años (de 1,9 a 3,5 autores por artículo).

Veamos algunos ejemplos:

La edición electrónica de la revista científica norteamericana Science, publica un dossier especial titulado «La secuencia del genoma humano» en la dirección http:// www.scienmag.org/genome200 I en el que presenta un extenso informe dé los resultados además de una serie de artículos que analizan las implicaciones de este avance científico desde el punto de vista médico, ético y legal. El artículo principal aparecido en Science está firmado por 263 investigadores encabezados por Craig Venter, fundador y director de Celera Genomics (http://www.celera.com). Entre 
las conclusiones publicadas están que todos los seres humanos comparten el 99,9 $\%$ de su secuencia genética. En el dossier hay otros artículos relacionados con el estudio del genoma, entre ellos, una comparación con los resultados del Proyecto Genoma Humano, el estudio de la expresión de los genes en tejidos cancerosos, y los retos de la proteómica, la nueva ciencia que ahora deberá estudiar la función de las proteínas en los procesos celulares, entre otros.

Para Bárbara R. Jasny y Donald Kennedy "la secuenciación del genoma humano representa, no un final, sino el principio de un nuevo acercamiento a la biología... hemos recibido una herramienta poderosa para desvelar los secretos de nuestra herencia genética y para encontrar nuestro lugar entre los otros participantes en la aventura de la vida". Pero los descubrimientos plantean lagunas jurídicas generadas por el nuevo conocimiento y los riegos de conocer las características genéticas de una persona. Otros apartados analizan qué significa la existencia de treinta mil genes en el hombre $y$, especialmente, las tareas pendientes, como distinguir los factores genéticos de los ambientales en la manifestación de las enfermedades y darle sentido a los datos recopilados hasta ahora es una nueva tarea de investigación en equipos.

Lo mismo sucede con los descubrimientos de la misión de la sonda Rosetta de la Agencia Espacial Europea (ESA) que lidera la lista de los diez "Descubrimientos del 20 I 4 elaborada por la revista "Science". Como se sabe Rosetta y su módulo Philae es el primer aparato enviado por el ser humano que aterriza sobre un cometa (67P/ Churyumov-Gerasimenko) sino por su aporte a la ciencia que permitirá realizar con los datos recogidos. Según Robert Coontz con sus datos "la mayor parte de la buena ciencia realmente está por venir". El reconocimiento de Science se suma al de otra revista especializada, Nature, que nombró ayer a Rosetta y al equipo detrás del proyecto como uno de los logros científicos del año.

Por otra parte el hallazgo paleontológico del año fue el cálculo correcto de la antigüedad de unas pinturas de animales en una cueva en Indonesia que se creía que fueron realizadas hace 10000 años, pero en realidad tenían entre 35000 y 40000 años, lo que apunta a que los humanos en Asia produjeron arte simbólico a la par que los europeos.

La publicación también reconoció una serie de artículos que compararon los fósiles de aves primitivas y dinosaurios con los pájaros modernos y permiten desvelar la evolución genética de las diferentes especies de aves. No sólo se logró el árbol genealógico más completo de las aves, que permite conocer detalles sobre su evolución explosiva tras la extinción de los dinosaurios -que dio origen a más de 10 mil especies-, sino que a mediados de año se conoció cómo los dinosaurios terópodos (una suborden carnívora y bípeda) fueron adquiriendo rasgos de aves, volviéndose más pequeños y livianos, un proceso que tardó 50 millones de años. 
Este proyecto internacional, en el que participaron más de 200 científicos, refuerza la teoría de un "big bang" de evolución de las aves durante los 10 a I 5 millones años que siguieron a la extinción de los dinosaurios y sugiere que el ancestro común más antiguo de las aves terrestres, que incluyen desde loros a halcones y águilas, era un superdepredador.

Asimismo, valoró un experimento que demostró que el factor GDFI I de la sangre de ratón joven puede rejuvenecer los músculos y el cerebro de ratones más viejos, y que ha llevado a un ensayo clínico en el que pacientes de Alzheimer reciben plasma de donantes jóvenes.

También entre los estudios destacados están, además, una investigación de la Universidad de Harvard que se inspiró en el comportamiento de las termitas para crear un grupo de robots que se coordinan entre si y son capaces de crear estructuras sin supervisión humana.

La lista también incluye otra investigación, con el uso de la optogenética una técnica que manipula la actividad neuronal con rayos de luz-, descubrimiento que permitió mostrar que es posible manipular recuerdos específicos en ratones.

En el área de neurociencia, destacaron los primeros chips "neuromórficos" que imitan la arquitectura del cerebro humano y están diseñados para procesar información de la forma más parecida a cómo lo hacen los cerebros vivos. El chip presentado por ingenieros de la compañía IBM es capaz de procesar información de manera similar a la del cerebro humano, con el chip, un procesador podría sentir, oler, escuchar y entender, eso ya que puede realizar más de un cálculo a la vez. La red cerebral humana tiene 100 mil millones de células vinculadas a 100 millones de millones de sinapsis (unión intercelular). TrueNorth, el chip de IBM, incluye 5,4 mil millones de transistores y 256 millones de sinapsis y la compañía trabaja por aumentarlos.

Dos investigaciones pioneras que desarrollaron dos métodos diferentes para cultivar células que se asemejan a las células beta -las células productoras de insulina del páncreas- también fueron reconocidas por su valía para dar a los investigadores una oportunidad "sin precedente" para estudiar diabetes. Las células Beta son las células del páncreas, que sintetizan y agregan la insulina, hormona que controla los niveles de glucosa en la sangre. La diabetes tipo I las destruye, impidiendo la producción de insulina. Por eso, desde el descubrimiento de las células madre, los científicos han tratado de convertir células adultas en Beta, para reemplazarlas en diabéticos y conseguir una cura. Este año, dos grupos científicos publicaron métodos para reprogramarlas y cultivarlas en laboratorio. Uno de ellos tarda siete semanas en convertirlas en productoras de insulina, en una cantidad suficiente para tratar un paciente. El próximo paso es hacer que el sistema autoinmune no las rechace. 
En el área de tecnología, sobresalieron los pequeños satélites conocidos como "Cubesats", pequeños satélites en forma de cubo, surgieron como una herramienta educacional para que estudiantes universitarios probaran su tecnología enviándola al espacio, operando un satélite similar al Sputnik (el primer satélite lanzado en 1957). Ahora, estas cajas de $10 \mathrm{~cm}$ comenzaron a hacer ciencia real, pueden tomar imágenes, enviar comunicaciones por radio y realizar monitoreo atmosférico, por ejemplo. Setenta y cinco fueron lanzados este año y están permitiendo que instituciones y países tengan acceso al estudio espacial, pues son mucho más accesibles y se pueden construir, incluso, siguiendo instrucciones por internet.

Así mismo se crean robots cooperativos a imitación del trabajo de hormigas y abejas, Radhika Nagpal, experta de la U. de Harvard, lideró uno de los equipos que logró que I.024 kilobots (robots pequeños y simples) se movieran y se comunicaran entre ellos, usando luz infrarroja, para crear formas de dos dimensiones. Otro proyecto, I 0 cuadricópteros se organizaron, ajustando sus caminos para no colisionar y volar creando un círculo rotatorio.

Por otra parte al igual que en la película Inception, donde un equipo es capaz de implantar un recuerdo en la mente de una persona, el 2013 científicos del Instituto Tecnológico de Masachussetts (MIT) lograron hacer lo mismo en ratones, en los que implantaron recuerdos falsos que impactaron en sus reacciones. Un año antes habían descubierto qué células son las que almacenan los nuevos malos recuerdos. Este año, el mismo equipo, liderado por Susumo Tonegawa, Nobel de Medicina 1987, probó que también es posible convertir los buenos recuerdos en malos o viceversa, manipulando las neuronas, al menos en ratones, pues aún es improbable hacerlo con humanos.

Mientras que en genética, destacaron unas bacterias sintéticas de E.coli que podrían ser utilizadas para crear proteínas de diseñador con aminoácidos "no naturales".

¿Cómo opera la transdisiciplinariedad en estos descubrimientos?

Vivimos una etapa de transición en la construcción de la ciencia bajo un nuevo paradigma de vida y conocimiento. Los descubrimientos antes señalados ubican un nuevo paradigma caracterizado por una epistemología múltiple y multidimensional de carácter integrador en su enfoque sistémico y enfoque histórico. Un modelo de modelos pos positivista que une o vincula el objeto con el sujeto de conocimiento en la unidad de sus diferencias sistémicas e históricas.

La visión fisicalista o naturalista del saber occidental que primó a lo largo de los siglos XIX y XX bajo la mirada cuantitativa instrumental y aplicativa se complementan hoy con la búsqueda de la explicación y la comprensión multicausal y relacional de los problemas de la vida y sus soluciones integrales. El desarrollo del mercado 
financiero global y los mercados de producción y consumo globales exigen la unidad del descubrir y el hacer. Ciencia, técnica y tecnología unidas en cada uno de los descubrimientos como nos muestran las investigaciones del genoma humano, cerebro humano, telescopio Hubble como el desarrollo de la tecno física, tecnogeología, tecnogeografía, tecnobiología, tecnoquímica, tecno ciencias sociales.

La lógica general epistémica y metodológica en los descubrimientos científicos se mantienen: preguntas, hipótesis, datos, teoría; pero la manera de descubrir las causas y las relaciones de los problemas se hace en equipos situación que altera la práctica del conocer y aplicar los conocimientos de la ciencia clásica moderna. Vemos predominar la tecno ciencia la cual cada vez más se ajusta por ahora a las necesidades e intereses de un mercado en creciente expansión global. Como anota un estudio esta revolución cientííca aplicada:

"I .- No alteran únicamente el conocimiento, sino ante todo la práctica científica y tecnológica. 2.- Las tecnociencias modifican el mundo social, no sólo la naturaleza. Lo principal es la transformación del mundo que producen, y en particular del mundo social. 3. Las revoluciones tecnocientíficas conllevan un profundo cambio en el lenguaje cientííco y tecnológico, pero dicha transformación no atañe a las relaciones de significado entre el lenguaje y la naturaleza, que son las que preocuparon a Kuhn. $\mathrm{S}$. En resumen, y para no detenernos excesivamente en este punto, las revoluciones tecnocientíficas conllevan un cambio de lenguaje muy importante, y en esto coincidimos con Kuhn. Pero los tecnolenguajes no son referenciales y tampoco se refieren a la naturaleza, al menos en primera instancia. Las referencias de los lenguajes informáticos no son objetos naturales, sino info-objetos. Los datos y las hipótesis se contrastan en ese nuevo espacio semiótico mediante simulaciones informáticas, modificación de parámetros, etc. La caracterización kuhniana de las revoluciones cientificas resulta insuficiente para las tecnocientíficas, porque los tecnolenguajes informáticos son de índole muy distinta a los lenguajes científicos clásicos.

En primer lugar, es claro que el rigor, la coherencia (interna y externa) y la simplicidad desempeñan un papel importante a la hora de valorar teorías, incluso cuando estas teorías son inconmensurables desde el punto de vista del conocimiento, es decir, en los casos de crisis de un paradigma y de revoluciones científicas. De lo dicho hasta ahora puede inferirse que el modelo kuhniano para las revoluciones científicas tiene que ser ampliado y modificado en varios puntos para intentar aplicarlo a las revoluciones tecnocientíficas. Kuhn distinguió entre las generalizaciones simbólicas, los modelos, los valores y los ejemplares de un paradigma.

La nueva estructuración de la práctica cientíica genera nuevos instrumentos formales que, conformen se convierten en estándares nacionales o internacionales, pueden ser considerados como generalizaciones simbólicas que ordenan la práctica científica misma. Vimos que las empresas tecnocientíficas son mucho más amplias y complejas que los observatorios, laboratorios y gabinetes de los científicos clásicos. Además de los protocolos de laboratorio y de los artículos para revistas científicas, los tecnocientíficos 
han de saber cumplimentar adecuadamente otro tipo de documentos: formularios para solicitar proyectos de investigación, informes de evaluación, hojas de cálculo, contratos de patentes, etc. Este segundo tipo de generalización simbólica no es científica, sino económica, administrativa y jurídica, pero ya vimos que la burocracia y la gestión son una parte fundamental de las empresas tecnocientíficas.

El sujeto que lleva a cabo las revoluciones tecnocientíficas no es un sujeto individual (como Einstein o Mendel), sino un conjunto de agentes sociales. Los vínculos que les mantienen unidos son diversos, pero la existencia de lazos estables y alianzas estratégicas entre gremios diversos (científicos, tecnólogos, políticos, empresarios, militares, etc.) son un factor indispensable para el progreso de una revolución tecnocientífica. Dichos vínculos son transdisciplinarios, a diferencia de las revoluciones científicas. Conforme a lo dicho en el párrafo anterior, incluyen agentes expertos en la comunicación del conocimiento a la sociedad, o cuando menos a sus dirigentes. De ahí la importancia crucial de la difusión y recepción de las innovaciones tecnocientíficas, que se manifiesta en el mercado, por una parte, pero también a nivel de opiniones y de actitudes de los diversos sectores sociales".

Los nuevos conocimientos integran la investigación básica, la investigación aplicada, el desarrollo tecnológico, la innovación, la producción, la comercialización, el consumo: vida, guerra (muerte), ganancia. Pero también se afirman en institucionalidades privadas y/o públicas como organizaciones sistémicas inteligentes. Por tanto como anota el estudio antes señalado:

"el conocimiento es un medio para la acción, no un fin en sí mismo. Por esa razón, las teorizaciones filosóficas sobre los objetivos de la ciencia no valen para la tecnociencia. La filosofía de la ciencia ha de cambiar porque ha cambiado la ciencia, y en particular sus objetivos. Incluso la búsqueda interminable de la verdad, por recordar al venerable Popper, se convierte en un instrumento para aumentar la capacidad de acción. Esta es la razón por la que venimos insistiendo en que la filosofía de la ciencia ha de centrarse en la actividad científica, más que en el conocimiento. Caso de haberla, lo cual habrá que estudiar a fondo, la racionalidad de la tecnociencia es práctica. Por ello resulta imprescindible optar por unos u otros modelos de racionalidad práctica, pero no en el sentido ético de la expresión (aunque la ética también desempeña un papel importante en los estudios sobre la tecnociencia), sino en el sentido de la acción racional. Ya hemos manifestado nuestras críticas a las concepciones instrumentales de la racionalidad, que siguen vigentes en la tecnociencia (el conocimiento es un medio), así como nuestra opción por la racionalidad valorativa o axiológica. El debate al respecto será largo y esta opción por la racionalidad acotada frente a la racionalidad maximizadora de la teoría de la decisión racional es un primer paso en esa dirección".

Una hegemonía creciente de la tecnociencia acelera la restructuración del sistema capitalista en organizaciones económicas financieras cada vez más transnacionales y multinacionales que se integran en alianzas económicos políticas diferenciadas y complementarias tomando como base el nuevo modo de desarrollo de la sociedad 
de la información y la comunicación para en su aplicación generando nuevos conocimientos y aplicaciones en innovaciones permanentes.

Esta situación lleva a la trans y multidisciplinaridad en toda su dimensión histórica y sistémica en la investigación de los problemas de la vida en su sentido aplicativo superando el pasado esquema positivista de la ciencia occidental eurocéntrica que separaba el sujeto del objeto, el conocimiento de la naturaleza y la sociedad. Un conocimiento multiparadigmático, multicausal y relacional con una visión organizacional y prospectiva del conocimiento de la realidad.

Vemos así enriquecerse la perspectiva interdisciplinaria en una perspectiva transdisciplinaria en el conocimiento filosófico cientíico teórico y cada vez más sus aplicaciones a la transformación de las situaciones problemáticas del mundo. Como destacan algunos estudios, si en siglo $X X$ el círculo de Viena con Otto Neurath, R. Carnap buscó unificar la solución de los problemas integrando los aspectos teóricos con los empíricos; el Centro de Educación Integral construye un pensamiento educativo con una visión general y holística; la teoría de sistemas con su enfoque relacional lo integra todo en el sistema cerrado u abierto; el constructivismo genético (Piaget), civilizatorio (Elias), social (Bourdieu), antropológico (Levi-Strauss), lingüístico (Barthes), institucional (Focault), objetos(Latour) entre otros científicos sociales fueron sentado los elementos para la construcción del nuevo paradigma científico.

Recordemos como resalta Jaim Royero para Piaget la interdisciplinaridad era: "la cooperación entre varias disciplinas o sectores heterogéneos de una misma ciencia... lleva a interacciones reales, es decir a una cierta reciprocidad de intercambios que dan como resultado un enriquecimiento mutuo". Algo similar a lo que señala Smimov: "La interdisciplinaridad en el campo de la ciencia consiste en una cierta razón de unidad de acciones y relaciones recíprocas, de interpretaciones entre las llamadas disciplinas científicas"

Este cambio de paradigma se da en un contexto global como destacan otros estudios como los de Souza Silva:

"En un nuevo régimen de acumulación del capital; el establecimiento de un Estado-red supranacional; la integración de políticas globales como la del desarrollo sostenible del planeta; y el nuevo modo de generación de conocimiento basado en la información y la comunicación. Un mundo como un mercado sin sociedades ni ciudadanos sino solo como consumidores. Una globalización que reemplaza los fines por los medios. Una intensa movilidad del capital y la creciente vulnerabilidad del trabajo. El desempleo tecnológico como fuente del lucro. La privatización de lo público. La creciente oposición entre el individualismo consumista y la solidaridad sustentable. El crecimiento económico con exclusión. El llenar el vacío de los valores con una nueva ética de responsabilidad. Las integraciones en redes y creciente fragmentación social y política. La emergencia de una ciencia sin consciencia marcada sólo por la 
lógica de la ganancia y no lo social. La discriminación genética por las ciencias de la vida. Un mundo del trabajo basado en la explotación intensiva por la razón del mercado. Una razón de mercado donde la realidad virtual manipula la realidad real. Un mercado "libre" que no es "libre". Una democracia representativa donde los electores no deciden y los que deciden no son electos. Un "buen gobierno" para las transnacionales bajo un pensamiento único e internacionalización creciente de la soberanía nacional. La permanente innovación bajo la creciente maquinización de la vida bajo nuevas integraciones y exclusiones, sociabilidades e individuaciones".

Como anota Latour un cambio socio tecnológico radical con relación a las otras revoluciones industriales donde: "En algún momento de la historia, las interacciones humanas se vieron mediadas por un cuerpo político amplio, estratificado y externo que seguía el rastro, gracias a una serie de «técnicas intelectuales»(básicamente escribir y contar), de los numerosos subprogramas de acción anidados. Cuando algunos de esos subprogramas, aunque no todos, quedan sustituidos por no humanos, nacen la maquinaria y las fábricas. Desde este punto de vista, los no humanos forman parte de una organización que ya está en funcionamiento y asumen un papel que durante siglos ha venido siendo desempeñado por una multitud de obedientes siervos humanos enrolados en la megamáquina imperial." 


\section{Bibliografía}

Aguayo, C. (2006). Las Profesiones Modernas: Dilemas del Conocimiento y del Poder. Santiago: Universidad Tecnológica Metropolitana.

Arnold, M. (1998). "Recursos para la investigación sistémico-constructivista". Santiago: Cinta de Moebio N ${ }^{\circ} 3$

Arnold, M., Cadena, H. y Urquiza, A. (20I4). La organización de las organizaciones sociales Aplicaciones desde perspectivas sistémicas. Santiago: Ril Editores.

Ballesteros, A. (2007). Max Weber y la sociología de las profesiones. México: UNPMax Textos. Véase versión electrónica.

Battistini, O. Bilakowsky, A. Busso, M. y Costa, M. (20I I). Los trabajadores en la nueva época capitalista. Buenos Aires: Editorial Teseo.

Bialakowsky, A. (2013). Coproducción e intelecto colectivo. Buenos Aires: Editorial Teseo.

Capel, H. ( 1 989). Historia de la ciencia e historia de las disciplinas científicas. Objetivos y bifurcaciones de un programa de investigación sobre historia de la geografía. Año XII, № 84. Barcelona: Geo crítica Cuadernos Críticos de geografía humana Universidad de Barcelona.

Castells, M. (1996). La era de la información. Vol. I. La sociedad Red. Barcelona: Alianza

Castells, M. (20/4). La dimensión cultural de Internet, en www.oei.orgCleaves, P. (1985). Las profesiones y el Estado: El caso de México. México: Colegio de México.

Casanova, P. (2004). Las nuevas ciencias y la humanidades. Mèxico: Anthropos-IIS.

Echevarría, J. (201 I). Filosofía de la ciencia. En http://es.slideshare.net/edelinbravo29/filosofa-de-la-ciencia-javier-echeverra-1995

Echevarría, J. (20 I0). De la filosofía de la ciencia a la filosofía de la tecnociencia. En http://revistas.um.es/daimon/article/view/I47/2 I

Fajnzylber, F. (1992). "Industrialización en América Latina. En Nueva sociedad.

González, P. (2004). Las nuevas ciencias y las humanidades De la Academia a la Política. Madrid: Anthropos-IIS-Editorial Complutense. 
González, P. (20 I 5). Crisis terminal del capitalismo o crisis terminal de la humanidad. ALAI AMLATINA, 07/05/20I 5.

Gonzales, B. (1996). Cultura y Tercer mundo I. Cambios en el Saber Académico. Venezuela: Nubes y Tierra.

Hierrezuelo, J. (20 | 4). La ciencia y la tecnología en la revolución industrial. En https:// sities. Google.com/

Latour, B. (1992). Ciencia en acción. Cómo seguir a los científicos e ingenieros a través de la sociedad. Barcelona: Labor.

Latour, B. y Woolgar, S. (1995). La vida en el laboratorio. La construcción de los hechos científicos. Madrid: Alianza.

Locke, J. (S/F) (1986). On the conducy of human understanding in The Works Basada en Locke, J. ( I 823). Evans, J. (Ed.)The woks od John Locke. Londres: Scientia Verlag Aalen. Locke, J. Pensamientos sobre educación. Madrid: Akal.

Medina, M. (2014). Tecnociencia, en http://ctcs.fsf.ub/prometheus/index.htm Editorial Pueblos Unidos.

Morin, E.(1999). Los siete saberes necesarios a la educación del futuro. París. Organización de las Naciones Unidas para la Educación, la Ciencia y la Cultura. UNESCO. Editorial du Seuil.

Morin, E. (1994). Introducción al pensamiento complejo. Barcelona: Editorial Gedisa.

Morin, E. y Anne B. Kern. (1993). Tierra Patria. Barcelona: Editorial Kairós.

Morin, E.(1992). El Método IV. Las ideas. Madrid: Editorial Cátedra.

Morin, E. (1983). El Método II. La vida de la vida. Madrid: Editorial Cátedra.

Morin, E. (1988). El Método III. El conocimiento del conocimiento. Madrid: Editorial Cátedra.

Morin, E. (1984). Ciencia con conciencia. Barcelona: Editorial Anthropos.

Morin, E. (|98|). El Método I. La naturaleza de la naturaleza. Madrid: Editorial Cátedra.

Polanyi, K. (200 I). La gran transformación. Bs. As: FCE. 
Pollard, S. (198I). La conquista pacífica. La industrialización de Europa 1760-1970. España: Prensas Universitarias de Zaragoza

Revista Science anuncia los diez logros científicos de 2014 en Tendencias http://www. latercera.com/noticia/tendencias/20 I 4/I 2/659-609332-9-revista-scienceanuncia-los-diez-logros-cientificos-de-20 I4.shtml

Revista Science elige a Rosetta como el avance científico del año enEmol.com - http://www.emol.com/noticias/tecnologia/2014 //2//8/695264/revista-science-elige-a-rosetta-como-el-avance-cientifico-del-ano.html

Rifkin, J. (20 I4): Liderando la tercera revolución industrial. En www.nodo50.org

Rodríguez, N. (2008). Manual de sociología de las profesiones. Barcelona: Universitat de Barcelona.

Rios, J. (200 I ).La sociología en San Marcos. Hacia una revolución teórica del quehacer sociológicos. Lima: JRB.

Rifkin, J. (20 I 5). Liderando la tercera revolución industrial.NEn www.nodo50.org

Soria-Silva, S. (20 I5). Sindicato, profesión y trabajo en el capitalismo. En http://pendientedemigracion.ucm.es/info/ec/jec l 0/ ponencias/6 | 4soriasilva.pdf

Soria-Silva, S. "Flexibilização, financeirização e liberalização: a santíssima trindade da nova ordem do capital". In: IV Colóquio Latino-Americano de Economistas Políticos: Anais. São Paulo, 2004.

Urrea, J. (2012). El origen de las profesiones desde Weber. En https://prezi.com/ soojx6t I svsu/el-origen-de-las-profesiones-desde-weber/

Wallerstein, I. (1976). The modern World System: Capitalist Agriculture and the origins of the European Word Economy in the Sixteenth Century. New York: Academic Press.

Wallerstein, I. (Coordinador). (1996). Abrir las ciencias sociales. México: Siglo XXI Editores.

Weber, M. (1994). La Ética protestante y el espíritu del capitalismo. México: Editorial Coyoacán.

Weber, M. (1985). El problema de la irracionalidad en las ciencias sociales. Madrid: Editorial Tecnos.

Weber, M. (1977). Economía y sociedad: esbozo de sociología comprensiva. 3ra reimp. I y |l volúmenes. México: FCE. 
Weber, M. (1944). Economía y Sociedad. Esbozo de sociología comprensiva. México: Editorial FCE.

\section{Direcciones electrónicas}

http://html. rincondelvago.com/avances-tecnicos-y-cientificos-de-la-segundarevolucion-industrial.html

www.epistemealsur.blogspot.com

http://www.fing.edu.uy/catedras/disi/ctysociedad/Revoluciones

http://www.lanacion.com.ar/I 435778-asi-trabaja-la-ciencia-hoy

http://www.monografias.com/trabajos57/edgar-morin/edgar-morin2.

http://www.monografias.com/trabajos I 5/ciencia-actual/ciencia-actual.

http://www.emol.com/noticias/tecnologia/20 I 4/I 2//8/695264/revista-scienceelige-a-rosetta-como-el-avance-cientifico-del-ano.html 